\title{
Schulsozialarbeit als Einzelhilfe
}

\section{Fallarbeit in der Schule als unterschätztes Regelangebot der Jugendhilfe}

\author{
STEFAN FISCHER \\ Stefan Fischer, Diplom-Sozialpäda- \\ goge $(\mathrm{FH})$, leitet beim Stadtjugend- \\ amt München die Abteilung \\ Kinder, Jugend und Familie. Als \\ Sachgebietsleiter für den Bereich \\ Schulsozialarbeit hat er den Aufbau \\ dieses Arbeitsfeldes in München \\ begleitet. Er ist zudem langjähriger \\ Referent für die Fortbildungen des \\ Bayerischen Landesjugendamtes \\ zum Thema "Jugendsozialarbeit an \\ Schulen".
}

stefan.fischer@muenchen.de

\author{
Das Arbeitsfeld Schulsozialarbeit wird vonseiten \\ der Jugendhilfe systematisch unterschätzt. \\ Denn Schulsozialarbeit kann nicht nur Defizite \\ der Schule beheben, sondern auch blinde \\ Flecken der Jugendhilfe beseitigen.
}

Das Arbeitsfeld der Schulsozialarbeit, das sich in Deutschland in den 1990er Jahren eher zögerlich in Projektform entwickelt hat, wurde in den darauffolgenden zehn Jahren kontinuierlich ausgeweitet und hat nun in den letzten zwei bis drei Jahren nochmal einen enormen Ausbau erfahren.

In vielen Kommunen ist Schulsozialarbeit ein Regelangebot für viele Schulen geworden, das an Stellenumfang teilweise bereits an die Bezirkssozialarbeit heranreicht. In der Wahrnehmung vonseiten der Jugendhilfe hat Schulsozialarbeit allerdings anscheinend den Projektcharakter bewahrt: Es fehlt die feste Einplanung in das System der Jugendhilfe. Die Kooperation mit der Bezirkssozialarbeit und mit den Hilfen zur Erziehung ist selten systematisch geregelt, obwohl die Aufgaben, gerade im Bereich der Einzelfallarbeit, sich überschneiden.

Der vorliegende Artikel macht Vorschläge für ein Profil der Schulsozialarbeit, das den Stärken dieses Arbeitsfeldes gerecht wird und ihm einen passenden Platz im Gefüge des Beratungssystems der Jugendhilfe zuweist. Es wird auch der Frage nachgegangen, ob und wie es gelingen kann, das Angebot der Schulsozialarbeit zur Entlastung der Hilfen zur Erziehung zu nutzen.

\section{Stand der Schulsozialarbeit}

Der 14. Kinder- und Jugendbericht spricht von 3.025 Personen für das Jahr 2010, die im Arbeitsfeld der Schulsozialarbeit tätig sind. (1)

Das Bildungs- und Teilhabepaket der Bundesregierung sieht für einen kleinen
Teil der entsprechenden Haushaltsmittel die Möglichkeit vor, diese pauschal, ohne Einzelfallzuordnung zu vergeben, u. a. für das Angebot von Schulsozialarbeit in den deutschen Kommunen. Dies hat in den letzten Jahren zu einem geschätzten Ausbau von weiteren ca. 3.000 Stellen geführt. Schätzungen zur Folge sind weitere ca. 3.000 Personen im Bereich der Schulsozialarbeit als Mitarbeiterinnen und Mitarbeiter der Schulverwaltung oder von Schulen tätig, führen dabei aber oft ähnliche Aufgaben durch wie Jugendhilfemitarbeiter.

Wenn all diese Angaben stimmen, nimmt das Arbeitsfeld Schulsozialarbeit im Bereich der Jugendhilfe mit 10.000 Beschäftigten (2) einen beachtlichen Anteil ein: Der aktuelle Kinder- und Jugendbericht spricht vergleichsweise von 9.500 Beschäftigten im Allgemeinen Sozialdienst, als der größten Organisationseinheit innerhalb des Jugendamtes. (3) Zum Vergleich: Rabb/Rademacker berichten in ihrer Bestandsaufnahme aus dem Jahre 1987, dass in nur 14,5 Prozent der Jugendämter einzelne Projekte - oft Einzelpersonen - der Schulsozialarbeit existieren (freie und öffentliche Trägerschaft). (4)

Die Zahlen in einigen Kommunen zeigen, dass der aktuelle Vergleich mit dem Allgemeinen Sozialdienst (ASD) durchaus realistisch ist: So arbeiten in München zur Zeit ca. 350 Beschäftigte im Bereich der Bezirkssozialarbeit, rund 200 arbeiten als Schulsozialarbeiterinnen und Schulsozialarbeiter. (5) In Nürnberg stehen ca. 100 ASD-Mitarbeiterinnen rund 80 Schulsozialarbeiterinnen gegenüber, in Dortmund ist das Zahlenverhältnis mit $150 \mathrm{zu} 140$ in 
etwa gleich und, um noch eine kleinere Kommune zu nennen: In Kempten gibt es ca. 20 Mitarbeiterinnen im Bereich der Bezirkssozialarbeit, 15 arbeiten als Schulsozialarbeiterinnen. (6)

Diese quantitative Annäherung an die Bezirkssozialarbeit und die enormen Steigerungsraten haben der Schulsozialarbeit allerdings bislang in der Fachdiskussion nicht sehr viel mehr Aufmerksamkeit beschert, fachlich fristet sie weiterhin eher eine Randexistenz, sie ist bislang das versteckte Angebot der Jugendhilfe in der Schule.

\section{Fachdiskussion blendet Jugendhilfe oft aus}

Seit den 1990er Jahren dreht sich die Fachdiskussion über Schulsozialarbeit um das Thema Kooperation Schule Jugendhilfe: Es geht um die Notwendigkeit eines Jugendhilfeangebotes in der Schule, um die partnerschaftliche Zusammenarbeit mit der Schule, um die Anerkennung von Schulsozialarbeit als gleichberechtigtem Partner, die Probleme der Kooperation in dem fremden System Schule, mangelnde Wertschätzung durch den Kooperationspartner u. Ä. Die Fachdiskussion beschäftigt sich erstaunlicherweise mehr mit dem
Thema Schule und der Zusammenarbeit mit Schule als mit der Zusammenarbeit mit der Jugendhilfe.

Im Übrigen hat die Fachdiskussion über die Schulsozialarbeit oft die Schulkritik als Ziel: Die einseitige Bildungsausrichtung der Schule, die begrenzten Handlungsmöglichkeiten von Schule, Lehrerinnen und Lehrer machen das Arbeitsfeld der Schulsozialarbeit notwendig und wichtig, so die gängige fachliche Argumentation. Auch der aktuelle Kinder- und Jugendbericht argumentiert in diese Richtung. Schulkritik ist allerdings wenig dazu geeignet ein eigenständiges Arbeitsfeld der Jugendhilfe zu legitimieren, insbesondere in Zeiten, in denen die Wirkungsorientierung eine entscheidenden Rolle für die Bewertung von Jugendhilfeangeboten einnimmt.

Angesichts dieser Ausrichtung der fachlichen Debatte ist es folgerichtig, dass einige Kommunen und Landkreise die Zuständigkeit für die Schulsozialarbeit eher bei den Schulen und Kultusministerien sehen (7): Wenn sich Schulsozialarbeit mit einem Defizit der Schule begründet, muss Schule dieses Angebot auch finanzieren, so die durchaus einsichtige Argumentation.

Im Blick auf die konkrete Lebenswelt der Kinder und Jugendlichen greift die- se Argumentation allerdings zu kurz. Schulsozialarbeit ist ein wesentlicher Faktor kommunaler Gestaltungsmacht im Bildungsbereich, sie ermöglicht der Jugendhilfe über die Arbeit mit ganzen Klassenverbänden präventiv alle Kinder und Jugendlichen zu erreichen. Die Verortung an der Schule verleiht diesem Angebot aber auch Einblicke und Kompetenzen in die Lebenswelt der Jugendlichen, die es zu einem überaus wertvollen Bestandteil der Beratung und Einzelfallarbeit innerhalb des Systems der Jugendhilfe machen.

Kurz gefasst: Schulsozialarbeit schließt nicht (oder jedenfalls nicht nur!) eine Lücke im System der Schule, sie schließt eine Lücke im System der Jugendhilfe!

Aufgrund dieser Ausrichtung hat sich die Fachdiskussion bislang noch wenig mit den spezifischen, methodischen Stärken von Schulsozialarbeit beschäftigt. Das Arbeitsfeld Schulsozialarbeit wird anscheinend vonseiten der Jugendhilfe systematisch unterschätzt.

Karsten Speck argumentiert zwar zu Recht, dass "Schulsozialarbeit als eine sozialpädagogische Leistung am Ort Schule über grundsätzlich keine anderen, sondern dieselben Methoden, wie auch die systematisch übergeordnete soziale Arbeit verfügt«. (8) Schulsozial-

\section{Personelle Ressourcen für die Einzelfallarbeit in der Schule}

Das Angebot der Schulsozialarbeit besteht aus gruppenbezogenen Angeboten (Projekte mit Klassen, Gruppenarbeit außerhalb des Unterrichts), Gremienarbeit in der Schule, stadtteilbezogene Arbeit, Schulveranstaltungen etc. und der Einzelfallarbeit. Die Einzelfallarbeit wiederum besteht nicht nur aus intensiver, längerfristiger Fallarbeit. Auch hier ist zu differenzieren. Ein Rechenbeispiel wird zur Zeit in München diskutiert, das helfen kann, den Umfang und die Bedeutung von Einzelfallarbeit von Schulsozialarbeit einzuschätzen:

Ein beträchtlicher Teil der Einzelfallarbeit von Schulsozialarbeit besteht aus kurzen Informations- und Beratungsgesprächen in der Pause, im Lehrerzimmer, im Gruppenraum, Flur oder Beratungszimmer. Es ist sinnvoll, hier zumindest eine grobe messbare Unterscheidung einzuführen. In München haben wir uns entschlossen, bis zu drei Gesprächen als Kurzberatung zu definieren. Gesprächsfolgen die darüber hinausgehen, werden als »Intensive schulspezifische Einzelfallarbeit der Schulsozialarbeit" gewertet.
Schulsozialarbeit verwendet ca. 50 Prozent der Arbeitszeit auf Einzelfallarbeit, bei einer Vollzeitstelle also ca. 20 Stunden. Nach unseren Erfahrungen und Statistiken entfallen von diesen 20 Stunden ca. 5 Stunden auf Kurzberatungen. Es verbleiben also noch ca. 15 Stunden (brutto) für die intensive schulspezifische Einzelfallarbeit. Wir gehen davon aus, dass die Schulsozialarbeit im Durchschnitt (!) ca. 1,5 Stunden pro Fall/ Woche aufwendet. Das würde bedeuten, sie kann, auch wieder im Durchschnitt, für ca. 10 Fälle die intensive schulspezifische Einzelfallarbeit übernehmen.

Wir schlagen vor, dass diese intensive Einzelfallarbeit sich durchschnittlich auf maximal ein halbes Jahr erstrecken sollte. Übersteigt der Zeitaufwand pro Woche bzw. die Laufzeit der Fallbearbeitung anhaltend und wesentlich die vorgegebenen Durchschnittswerte, so sollte eine Übergabe an die Hilfen zur Erziehung, insbesondere an die ambulanten Erziehungshilfen angestrebt werden. Die Schulsozialarbeit arbeitet in diesen Fällen selbstverständlich weiterhin sowohl mit dem Kind oder Jugendlichen als auch den Hilfen zur Erziehung zusammen.
Voraussetzung für diese Funktion der Schulsozialarbeit ist allerdings eine angemessene Personalausstattung insgesamt. Die gruppenbezogenen und schulbezogenen Anteile der Schulsozialarbeit sollen keinesfalls verlorengehen, das fachliche Profil und die Vielseitigkeit des Arbeitsfeldes müssen erhalten bleiben. Die Stärke des Profils der Einzelfallarbeit der Schulsozialarbeit gründet sich auf ihre gruppenbezogene Arbeitsweise - ohne diese wird sie zum Allgemeinen Sozialdienst an der Schule.

In München rechnen wir für die Mittelschulen 17 Stunden pro 100 Schüler (d. h. ca. 1,5 Stellen) für eine Schule durchschnittlicher Größe, an den Grundschulen je nach Größe zwischen 30 und 50 Stunden pro Woche. Sollte die stärkere Akzentuierung der Einzelfallarbeit dazu führen, dass die vorgesehenen 50 Prozent für diesen Arbeitsanteil überschritten werden, werden wir an den entsprechenden Schulen die Möglichkeit eines weiteren Ausbaus prüfen, gegebenenfalls auch mit Mitteln aus dem Bereich der Erzieherischen Hilfen.

Stefan Fischer 
arbeit übernimmt Methoden der offenen Kinder- und Jugendarbeit, der Hilfen zur Erziehung und der Jugendsozialarbeit und passt sie den besonderen Bedingungen, die der Arbeitsort Schule bietet, an. Diese Anpassung an Schule als einen Teil der Lebenswelt von Schülerinnen und Schüler macht aber gerade die besondere Stärke dieses Arbeitsfeldes aus.

Schulsozialarbeit hat sich in den letzten Jahren als Bestandteil der Lebenswelt von Kindern und Jugendlichen in der Schule etabliert und dabei Kenntnisse und Kompetenzen erworben, die sie von allen anderen Angeboten der Jugendhilfe unterscheidet. Insbesondere im Bereich der Einzelfallhilfe von Schulsozialarbeit kommt dies zum Tragen.

\section{Indikatoren der Einzelfallhilfe in der Schulsozialarbeit}

Die Einzelfallhilfe ist der Arbeitsanteil der Schulsozialarbeit, der in der Regel zuerst genannt wird. Viele Konzepte gehen davon aus, dass Schulsozialarbeit zumindest zu einem Drittel aus Einzelfallarbeit besteht, oft wird von einem Anteil der Einzelfallarbeit von mindestens 50 Prozent geredet. Auch staatliche Förderkonzepte betonen die Tatsache, dass die Einzelfallarbeit der entscheidende Aspekt für die Wahrnehmung von Jugendhilfeverantwortung ist und den Kern des Angebots bilden muss. (9)

Viele Jugendhilfeangebote, vielleicht die meisten, arbeiten (auch) einzelfallorientiert. Die Schulsozialarbeit aber kann als einziges Jugendhilfeangebot ihre Einzelfallorientierung damit verbinden, dass sie an der Schule verortet ist, einen immer wichtiger werdenden Teil der Lebenswelt von Kindern und Jugendlichen. Dies verschafft ihr - als Strukturvorteil - Bezüge, Kenntnisse, Einblicke in diese Lebenswelt, die andere Angebote der Jugendhilfe wie beispielsweise die ambulanten Erziehungshilfen, sich mühsam methodisch erarbeiten müssen.

Das folgende Raster soll dazu dienen, Indikatoren dafür herauszuarbeiten, in welchen Fällen sich Schulsozialarbeit besonders dazu eignet, eine Einzelfallhilfe für ein Kind oder einen Jugendlichen zu übernehmen.

- Leichter Zugang - Beziehungsorientierung: Schulsozialarbeit ist innerhalb der Schule in der Regel bestens bekannt. Die betreffende sozialpädagogische Fachkraft kennt alle Lehrerinnen und Lehrer, viele Eltern, ist den Schülerinnen und Schülern zumindest vom Sehen her bekannt. Durch Gruppenangebote, Projekte mit Klassen, Veranstaltungen in der Schule, informelle Gespräche auf dem Pausenhof, entwickeln darüber hinaus viele Schülerinnen und Schüler auch eine gute Beziehung und ein Vertrauensverhältnis zur Schulsozialarbeit. Die Schulsozialarbeit ist darüber hinaus leicht, ohne große Hemmschwellen zu erreichen: Schülerinnen und Schüler, aber auch Eltern können ohne Voranmeldung die Schulsozialarbeit im Büro ansprechen, aber auch bei anderen Gelegenheiten den Kontakt suchen, eine erste Bekanntheit herstellen, bevor sie sich entscheiden, ob sie der Schulsozialarbeit ein Anliegen oder ein Problem anvertrauen. Schulsozialarbeit ist insofern gut geeignet für Kinder und Jugendliche, die sonst kaum erreichbar sind, die den Kontakt mit dem Jugendamt skeptisch gegenüberstehen, die schwer Vertrauen fassen, die nicht selbst um Unterstützung nachsuchen. Gleiches gilt für Eltern, die ungern an das Jugendamt herantreten, die den Kontakt zu Behörden eher scheuen. Für all diese Fälle ist die Schulsozialarbeit ein leicht zu- auch für Disziplin und Verhaltensprobleme in der Schule. Sie kann so hervorragend als Vermittler dienen zwischen der Lebenswelt von Kindern und Jugendlichen und der Schulwelt: Sie kann Lehrerinnen und Lehrer, Eltern, Schülerinnen und Schüler gleichermaßen verstehen, vermitteln, dazu beitragen, gemeinsame pädagogische Strategien zu finden. Schulsozialarbeit ist deshalb bestens geeignet für Fälle, in denen Kinder und Jugendliche oder Eltern Probleme mit der Schule haben. Schulprobleme, die häufig ihre Ursache in Verhaltensproblemen haben, sind aber mit einer der häufigsten Gründe für die Beantragung von Hilfen zur Erziehung, insbesondere von ambulanten Erziehungshilfen. Gerade in solchen Fällen könnte und sollte Schulsozialarbeit eine gute Alternative sein.

- Zugang zu Peergroup: Schulsozialarbeit erlebt die Kinder und Jugendlichen täglich mit Gleichaltrigen. Sie kann so zum einen deren Entwicklungsstand gut vergleichen, zum anderen kann sie aber auch wahrnehmen, wie Kinder und Jugendliche sich in Gruppen verhalten. Das Gruppenverhalten von Kindern und Jugendlichen in der Freizeit ist oft anders, als dies Eltern vermuten, unterscheidet sich auch vom Verhalten in der Klasse, die ein Lehrer beaufsich-

\section{"Die Verortung an der Schule verleiht der Jugendhilfe wertvolle Einblicke in die Lebenswelt der Jugendlichen"}

gänglicher Kontaktpartner, aus kurzen Einzelgesprächen kann sich eine konstante Beziehung und eine längerfristige Einzelfallarbeit entwickeln.

- Schulkompetenz: Die Schulsozialarbeit hat beste Kenntnisse über die Schulwelt, sie arbeitet eng mit Schule, Schulleitung, Lehrkräften, anderen schulischen Beratungsdiensten zusammen. Sie kennt gut das Regelsystem der Schule, Wertvorstellungen und Organisationskultur der Schule sind ihr vertraut. Schulsozialarbeit kann von daher schulische Probleme von Kindern und Jugendlichen gut einschätzen und einordnen. Dies gilt sowohl für Lernschwierigkeiten, als tigt. So kann die Schulsozialarbeit Kinder und Jugendliche zum einen sicher und realistisch einschätzen, zum anderen kann die Schulsozialarbeit die gleichaltrige Gruppe als Methode einsetzen. Kontaktscheue und schüchterne Kinder und Jugendliche können angesprochen und gezielt in eine Gruppe integriert werden, mit unangepassten und aggressiven kann gezielt daran gearbeitet werden, dass sie gruppenfähig werden. Schülerinnen und Schüler, die im Schulalltag wenig Erfolgserlebnisse haben, wenig Wertschätzung erfahren, kann dadurch geholfen werden, dass ihnen in gleichaltrigen Gruppen eine Funktion 
zugewiesen wird, in der sie ihre Stärken einbringen können. Schülerinnen und Schüler, die vielleicht selten eine gute Note zustande bringen, können trotzdem vielleicht in hervorragender Weise ein Kickerturnier organisieren, Fahrräder reparieren oder einen guten Beitrag zum Schulfest erbringen. Schulsozialarbeit kann insofern Fälle übernehmen, in denen es um die Integration in gleichaltrige Gruppen geht. Dies sind beispielsweise Fälle, in denen Eltern sich an das Jugendamt wenden, weil sie besorgt sind, dass ihre Kinder zu aggressiv sind, zu schüchtern sind, keine Freunde finden, in falscher Gesellschaft sind etc.

- Flexibilität: Die Schulsozialarbeit kann ihren Kontakt zu Schülerinnen und Schüler flexibel nach der jeweiligen Situation und Problemlage gestalten. In Einzelfällen ist es durchaus möglich, täglichen Kontakt zu pflegen oder wöchentlich Gespräche zu führen. Aber auch jedes andere Arrangement ist im Schulkontext leicht möglich: feste Kontakte an bestimmten Tagen, in bestimmten Pausen, nach Schulschluss, vor der Schule, festgelegte gemeinsame Gespräche mit Mitschülerinnen und Mitschülern, Lehrerinnen und Lehrern, Eltern usw. All diese Gesprächsangebote sind für die Beteiligten ohne organisatorische Schwierigkeiten, ohne Fahrtzeiten, leicht realisierbar. Darüber hinaus kann die Schulsozialarbeit die Einzelfallhilfe einfach und flexibel ergänzen durch Gruppenangebote: Sie kann der Schülerin oder dem Schüler eine Teilnahme an bestehenden Gruppen anbieten, kann für bestimmte Schülerinnen und Schüler oder Schülergruppen maßgeschneidert ein Angebot im Schulkontext konzipieren. Schulsozialarbeit eignet sich diesbezüglich für Fälle, in denen die Problemlage und der Bedarf nicht oder noch nicht überschaubar ist. Sie kann den Auftrag übernehmen, prozesshaft und bedarfsorientiert eine Einzelfallstrategie zu entwickeln.

- Kinderschutz: Die Diskussion über die notwendige Verstärkung des Kinderschutzes in Deutschland, die Einführung des $\mathbb{S} 8$ a SGB VIII und die Einführung des neuen Bundeskinderschutzgesetzes haben der Einzelfallhilfe eine neue, nicht immer erfreuliche Aufmerksamkeit verschafft. Die Diskussion hat zu einem erheblichen Ausbau entsprechender Unterstützungsangebote, insbesondere der Bezirkssozialarbeit und der Hilfen zur Erziehung (10) geführt. Die Schulsozialarbeit wird in diesem Zusammenhang bisher zu wenig erwähnt. Sie ist jedoch das Arbeitsfeld der Jugendhilfe, welches durch den unmittelbaren Kontakt oder über die Lehrerinnen und Lehrer oft als erstes Kenntnis von Gefährdungen erhält. Die Rolle der Schulsozialarbeit im Kinderschutz wird so sicher noch unterschätzt. Die Schulsozialarbeit als Regelangebot der Jugendhilfe eignet sich sehr gut als ihr präventives Frühwarnsystem, kann mögliche Gefahren für die Entwicklung von Kindern und Jugendlichen erkennen bevor daraus ein »Gefährdungsfall « wird. Die Zuständigkeiten und Kommunikationswege für die Vorgehensweise in einem Gefährdungsfall, die Zusammenarbeit von Schule, Schulsozialarbeit und Bezirkssozialarbeit, sind allerdings detailliert zu regeln. Dabei kann die Tatsache, ob die Schulsozialarbeit in öffentlicher oder freier Trägerschaft liegt, von Bedeutung sein.

- Fallzugang: Schulsozialarbeit »entdeckt" viele Fälle im schulischen Kontext selbst. Bei diesen Fällen entscheidet Schulsozialarbeit, ob und zu welchem Zeitpunkt sie mit anderen Beratungssystemen der Jugendhilfe zusammenarbeitet: den Erziehungsberatungsstellen beispielsweise, insbesondere aber mit der Bezirkssozialarbeit. In diesen Fällen entlastet sie das Einzelfallsystem der Jugendhilfe, das Beratungsangebot der Hilfen zur Erziehung, ohne das der Fall im Bereich der Hilfen zur Erziehung je in Erscheinung tritt. Die Schulsozialarbeit kann und soll jedoch auch von den anderen Hilfesystemen gezielt als Einzelfallhilfe mit besonderen Stärken für besondere Fallkonstellationen eingesetzt werden. So sollte im Rahmen eines Beratungsgespräches der Bezirkssozialarbeit, eines Beratungsangebotes einer Erziehungsberatungsstelle, aber auch im Rahmen eines Hilfeplanverfahrens durchaus auch die Schulsozialarbeit als die besser geeignete Hilfe vorgeschlagen werden.

\section{Entlastung für die Hilfen zur Erziehung}

Eine Initiative der Stadt Hamburg hat seit 2011 eine bundesweite Debatte über die Weiterentwicklung der Hilfen zur Erziehung ausgelöst: »Erzieherische Unterstützung im Einzelfall sollte nicht mehr vorrangig durch hochspezialisierte, teure und kaum steuerbare Einzelfallhilfen geleistet werden, sondern im Regelfall durch eine verbindliche Ausgestaltung ortsnaher, alltagsentlastender und unterstützender Infrastruktur im Sozialraum.« (11)

Der Beschluss der Jugend- und Familienministerkonferenz greift diese Debatte auf und fordert u. a. eine Stärkung der Regelstrukturen und von Prävention, insbesondere aber eine Verbesserung der Zusammenarbeit der Jugendhilfe mit dem Bildungssystem. (12) Es liegt nahe, sich bei dieser Debatte gerade an die Schulsozialarbeit zu erinnern: Die beschriebenen Stärken, ihre Nähe zu den Kindern und Jugendlichen tragen dazu bei, dass sie entlastend für die Hilfen zur Erziehung eingesetzt werden können.

Die Wirkungen von Schulsozialarbeit sind allerdings durchaus zwiespältig und der Entlastungseffekt ist nicht immer sofort nachweisbar: die Schulsozialarbeit »entdeckt«, wie ausgeführt, auch frühzeitig Fälle, sieht Unterstützungsbedarf bevor ein Fall aktenkundig wird. Durch eigene Interventionen oder frühzeitige Vermittlung anderer Hilfen kann sie aber intensivere kostspieligere Hilfen verhindern. Eine entsprechende Untersuchung in Karlsruhe scheint dies zu bestätigen: »Bei stark steigender Anzahl von Beratungsfällen (82\%) sind die Gesamtkosten von Beratungshilfen, Hilfen zur Erziehung und Schulsozialarbeit ... im Vergleich zum vorgehenden Schuljahr ... um $20 \%$ gefallen. (13)

\section{Fazit}

Schulsozialarbeit hat sich in den meisten Kommunen zu einem Regelangebot der Jugendhilfe entwickelt. Sie kann und soll verantwortlich Einzelfallarbeit im Beratungssystem der Kinder und Jugendhilfe übernehmen. Sie unterscheidet sich in ihrem Profil, ihren Stärken, ihrer Verortung ganz wesentlich von anderen Beratungsangeboten der Jugendhilfe. Sie soll diesen Stärken 
entsprechend eingesetzt werden. Schulsozialarbeit ist nicht der preiswerte Ersatz für eine Hilfe zur Erziehung. Sie kann diese ergänzen, ist oft aber oft auch - frühzeitig eingesetzt - die bessere, kompetentere und passendere Alternative.

\section{Anmerkungen}

(1) 14. Kinder- und Jugendbericht, S. 330 .

(2) Entwurf »Empfehlung für die Entwicklung und den weiteren Ausbau der Schulsozialarbeit ", Deutscher Verein für öffentliche und private Fürsorge, erscheint voraussichtlich Ende 2013.

(3) 14. Kinder- und Jugendbericht, S. 292. Bei dem Vergleich ist zu berücksichtigen, dass der ASD in den Ämtern unterschiedliche Aufgaben, nicht allein Jugendhilfe-Aufgaben wahrnimmt.

(4) Raab/Rademacker, Handbuch Schulsozialarbeit 1987, S. 259.

(5) Zum Vergleich: In München hat die Schulsozialarbeit im Jahre 1993 begonnen mit vier Personen, 1998 waren ca. 20 Fachkräfte in dem Arbeitsfeld tätig.

(6) Angaben der jeweiligen Jugendämter.

(7) Bayerischer Landkreistag/Bayerischer Städtetag.

(8) Speck, Karsten, Schulsozialarbeit, Eine Einführung, München, 2007, S. 63.

(9) Richtlinie zur Förderung der Jugendsozialarbeit an Schule, Bayerisches Staatsministeriums für Arbeit und Soziales, 2012, Handbuch zur Jugendsozialarbeit an Schulen in Bayern, München 2004, S. 27.

(10) 4. Kinder- und Jugendbericht, S. 296.

(11) Kurz-Adam, Maria, Die Sorge um das Subjekt. Anmerkungen zur aktuellen Steuerungsdebatte in der Kinder- und Jugendhilfe, Neue Praxis, 6/2011, S. 571.

(12)Jugend- und Familienministerkonferenz, Beschluss vom 31.5./ 1.6.2012 zur Weiterentwicklung und Steuerung der Hilfen zur Erziehung.

(13) Niederbühl, Reinhard, Wirksamkeit und Effizienz von Schulsozialarbeit, in Speck/Olk, Forschung zur Schulsozialarbeit, S. 305 f.

\section{Literatur}

Bayerisches Staatsministerium für Arbeit und Sozialordnung, Familie und Frauen: Handbuch zur Jugendsozialarbeit an Schulen in Bayern,

München 2004.

Bayerisches Staatsministerium für Arbeit und Sozialordnung, Familie und Frauen: Richtlinie zur Förderung der Jugendsozialarbeit an Schulen - JAS, vom 20.11.2012.

Bundesministerium für Familie, Senioren, Frauen und Jugend: 14 . Kinder- und Jugendbericht, Berlin, 2013.

Jugend- und Familienministerkonferenz,

Beschluss vom 31.5./1.6.2012 zur Weiterentwicklung und Steuerung der Hilfen zur Erziehung.

Kurz-Adam, Maria, Achtsame Hilfen, eev-

aktuell, 2012

Kurz-Adam, Maria, Was brauchen Kinder? Perspektiven für die Erziehungshilfen und die Kinder- und Jugendhilfe, München 2012.

Kurz-Adam, Maria, Die Sorge um das SubjektAnmerkungen zur aktuellen Steuerungsdebatte in der Kinder- und Jugendhilfe, Neue Praxis, 6/2011

Frommann, Kehrer, Liebau: Erfahrungen mit Schulsozialarbeit, Weinheim und München 1987. Olk, Bathke, Hartnuss: Jugendhilfe und Schule, Weinheim und München 2000

Raab, Rademacker, Winzen: Handbuch Schulsozialarbeit, Deutsches Jugendinstitut München, 1987.

Speck, Olk: Forschung zur Schulsozialarbeit, Weinheim und München 2010.

Speck, Karsten, Schulsozialarbeit, Eine Einführung, München, 2007.

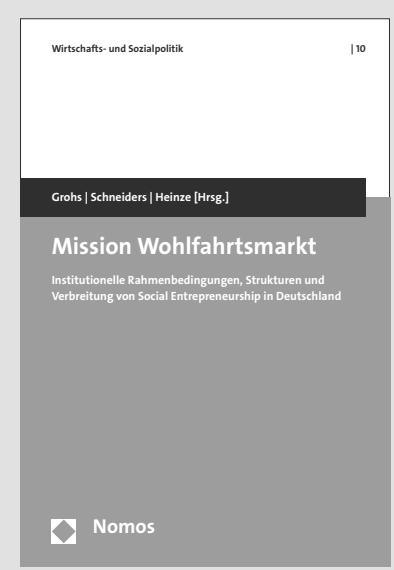

Mission
Wohlfahrtsmarkt Institutionelle Rahmenbedingungen, Strukturen und Verbreitung von Social Entrepreneurship in Deutschland Von AR Dr. Stephan Grohs, Prof. Dr. Katrin Schneiders und Prof. Dr. Rolf G. Heinze

2013, ca. 220 S., brosch., ca. 39,-€ ISBN 978-3-8329-7874-7

(Wirtschafts- und Sozialpolitik, Bd. 10)

Erscheint ca. Dezember 2013

Mit Social Entrepreneurship ist ein (vermeintlich) neuer Trägertyp entstanden, der durch die Verknüpfung von sozialem Engagement und unternehmerischem Handeln die Effektivität sozialer Dienstleistungen zu verbessern sucht. Das Buch bietet auf Grundlage einer Befragung von ca. 2.000 Organisationen erstmalig eine umfassende Analyse dieses neuen Phänomens.

www.nomos-shop.de/19785

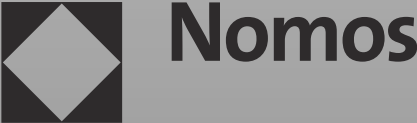

